\title{
Erythropoietin Improves Mood and Modulates the Cognitive and Neural Processing of Emotion 3 Days Post Administration
}

\author{
Kamilla Miskowiak*,1,2, Becky Inkster ${ }^{2}$, Sudhakar Selvaraj ${ }^{2}$, Richard Wise ${ }^{3}$, Guy M Goodwin ${ }^{2}$ and \\ Catherine J Harmer ${ }^{1,2}$ \\ 'Department of Experimental Psychology, University of Oxford, Oxford, UK; ${ }^{2}$ Department of Psychiatry, Warneford Hospital, University of \\ Oxford, Oxford, UK; ${ }^{3}$ Department of Clinical Neurology, FMRIB Centre, John Radcliffe Hospital, Oxford University, Oxford, UK
}

\begin{abstract}
Erythropoietin (Epo) has neuroprotective and neurotrophic effects and is a promising candidate for treatment of neurodegenerative and psychiatric disorder. Recently, we demonstrated that Epo modulates memory-relevant hippocampal response and fear processing in human models of antidepressant drug action I week post-administration, and improves self-reported mood for 3 days immediately following administration. The present study explored the effects of Epo (40000 IU) vs saline on self-reported mood and on neural and cognitive function in healthy volunteers 3 days post-administration to test the reliability of the rapid mood improvement and its neuropsychological basis. Neuronal responses during the processing of happy and fearful faces were investigated using functional magnetic resonance imaging ( $\mathrm{fMRI}$ ); facial expression recognition performance was assessed after the $\mathrm{fMRI}$ scan. Daily ratings of mood were obtained for 3 days after Epo/saline administration. During faces processing Epo enhanced activation in the left amygdala and right precuneus to happy and fearful expressions. This was paired with improved recognition of all facial expressions, in particular of low intensity happiness and fear. This is similar to behavioral effects observed with acute administration of serotonergic antidepressants. Consistent with our previous finding, Epo improved self-reported mood for all 3 days post-administration. Together, these results suggest that characterization of the effects of Epo in a clinically depressed group is warranted.

Neuropsychopharmacology (2008) 33, 6I I-6I8; doi: I0.1038/sj.npp. I301439; published online 2 May 2007
\end{abstract}

Keywords: Erythropoietin; mood; emotion; memory; fMRl; healthy volunteers

\section{INTRODUCTION}

While conventional drug treatments of depression are effective for many patients, there remains a subset for which these treatments are ineffective. Current drug treatments are also limited by slow clinical action and unwanted side effects. Hence, there remains a real need for novel treatment strategies, which may work via different mechanisms or which have more rapid antidepressant effects. There is particular interest in the hypothesis that neural atrophy and reduced neurogenesis are involved in the pathophysiology of depression while increased cellular resilience and plasticity are key mechanisms in chronic antidepressant treatment (Santarelli et al, 2003; Berton and Nestler, 2006). This neurotrophic hypothesis is supported by evidence that increased hippocampal brain-derivedneurotrophic-factor (BDNF) is necessary for the behavioral effects of antidepressant treatment (Santarelli et al, 2003)

*Correspondence: K Miskowiak, Department of Psychiatry, Warneford Hospital, University of Oxford, Oxford OX3 7JX, UK, Tel: + 4401865 233612, Fax: + 4401865251076

E-mail: kamilla.miskowiak@psy.ox.ac.uk

Received 2 February 2007; revised 27 March 2007; accepted 29 March 2007 and that infusion of BDNF into the hippocampus mimics the behavioral effects of antidepressants in rodents (Shirayama et al, 2002). Notably, the antidepressant effects of BDNF infusion were observed as early as 3 days postadministration and lasted for at least 10 days (Shirayama et al, 2002).

Erythropoietin (Epo) is used for the treatment of anemia associated with chronic renal failure and chemotherapy. Epo has recently received attention for neurobiological actions mediated through a non-hematopoietic Epo receptor system in the central nervous system (CNS) of animals and man (for review, see Brines and Cerami, 2005). Systemically administered Epo crosses the blood-brain barrier and has neuroprotective and neurotrophic effects in experimental CNS damage (Brines et al, 2000) and animal models of neurodegenerative and neuropsychiatric conditions (Brines and Cerami, 2005). Evidence from animal models suggests that these actions are mediated through several mechanisms including rapid upregulation of BDNF (Wang et al, 2004; Viviani et al, 2005) and neurogenesis (Wang et al, 2004), downregulation of neural apoptosis in brain regions adjacent to primary damage site (Siren et al, 2001), and axonal regeneration after axotomy (King et al, 2007). In humans, Epo commenced within $8 \mathrm{~h}$ post-stroke reduces infarct size and improves neurological recovery 
after 1 month (Ehrenreich et al, 2002). In addition, weekly administration of Epo vs saline to schizophrenic patients improved cognitive function after 12 weeks (Ehrenreich et al, 2007). However, because of concomitant upregulation of red cell mass in schizophrenic patients treated with Epo $v s$ placebo, it is unclear whether this effect was mediated by the direct neuroprotective actions or increased oxygen delivery to the brain. Similarly, earlier studies have found a correlation between elevation of red blood cell levels and improved cognitive function, mood, and life quality in anemic patients treated with Epo (Nissenson, 1992; Leitgeb et al, 1994) implying that the beneficial effects of Epo may be non-specific and due to correction of anemia (Nissenson, 1992).

Given this background, we previously assessed the effects of a single dose of Epo on neural and cognitive function in human models of antidepressant drug action independent of any hematological effects (Miskowiak et al, 2007). One week post-administration, Epo downregulated occipitoparietal blood-oxygen-level dependent (BOLD) response to fearful $v s$ neutral faces and reduced recognition of fearful expressions, similar to the effects of serotonergic and noradrenergic antidepressants (Harmer et al, 2004, 2006). In addition, Epo enhanced the hippocampal BOLD response during picture retrieval (Miskowiak et al, 2007), perhaps consistent with increased hippocampal BDNF and neurogenesis (Hariri et al, 2003). Unexpectedly, Epo also improved self-reported mood for 3 days post-administration, reflected by reduced negative affect scores on the positive and negative affect scales (PANAS), indicating increased calmness and serenity (Watson et al, 1988). Although many studies do not find effects of antidepressants on conventional measures of mood in healthy volunteers (eg Harmer et al, 2004, 2006), one study has reported decreased negative affect scores after an SSRI $v s$ placebo in non-depressed participants (Knutson et al, 1998).

The aim of the present study was to investigate this rapid mood change by assessing the accompanying neuronal and psychological actions of Epo at this early time point. The acute mood improvement following Epo led us to hypothesize that this group would show neuronal evidence of increased positive emotional processing relative to placebo treatment.

\section{METHODS}

\section{Participants}

Ethical approval for the study was obtained from the Oxfordshire Research Ethics Committee. Healthy volunteers were screened through a medical examination and psychiatric interview using the Structured Clinical Interview for DSM-Clinical Version (SCID-IV). Exclusion criteria were: history of psychiatric disorder, any significant medical condition (including diabetes, epilepsy, hypertension, and thrombosis), pregnancy, current medication (including the contraceptive pill), or first-degree family history of blood clotting, heart disease, or seizure disorders. Functional magnetic resonance imaging (fMRI) scanning also required the following exclusion criteria: cardiac pacemaker, mechanical heart valve or any mechanical implants. A baseline blood sample was taken to check that hematocrit, hemoglobin $(\mathrm{Hb})$, renal function, liver function, and ferritin levels were normal, and a pregnancy test was performed on female volunteers. After complete description of the study to the participants, written informed consent was obtained.

\section{Experimental Design}

Twenty-four right-handed volunteers were randomly allocated to receive an intravenous injection of either Epo (40 $000 \mathrm{IU}$ ) or saline over $5 \mathrm{~min}$ in a randomized doubleblind between-groups design. Groups were matched for: gender (seven males per group), age (mean \pm SD: $23.3 \pm 4.7$ years and $23.7 \pm 6.2$ years, respectively), and IQ measured with the National Adult Reading Test (mean \pm SD of test score: $114.6 \pm 3.9$ and $113.3 \pm 4.7$, respectively). Blood pressure and subjective state were monitored for $2 \mathrm{~h}$ following the injection. Daily ratings of mood were obtained for 3 days after drug administration. fMRI scanning and psychological testing were performed on day 3. Blood pressure was measured at the beginning of the test day as well as immediately before and after scanning while volunteers were lying on the scanner bore. A follow-up blood sample was taken to check for any changes in levels of red cell mass.

\section{Experimental Paradigm}

During fMRI, tasks were projected from a computer using e-prime software (version 1.0; Psychology Software Tools Inc., Pittsburgh, PA) onto an opaque screen at the foot of the scanner bore. Participants viewed the screen through angled mirrors and responded by pressing the keys of a response pad with their thumbs. Thereby, accuracy and response time (RT) could be investigated.

Stimuli pictures of happy, fearful, and neutral faces acquired from the Pictures of Affect Series (Ekman and Friesen, 1976) were presented covertly and overtly in a blocked paradigm. The covert condition consisted of four blocks each of happy, fearful, and neutral faces presented for $17 \mathrm{~ms}$ each and immediately replaced by a neutral face mask for $183 \mathrm{~ms}$. Mask images were faces different from target faces but of the same gender. The overt condition contained four blocks each of fearful, happy, and neutral faces presented for $200 \mathrm{~ms}$. There were a total of 24 stimulus blocks, each $20 \mathrm{~s}$ long and consisting of 10 faces/face mask pairs. Stimulus blocks were presented in random order interspersed with $20 \mathrm{~s}$ of a fixation cross leading to a total task time of $16 \mathrm{~min}$. During this time, participants performed a simple gender discrimination task.

\section{Visual Stimulation Paradigm}

A control visual stimulation paradigm was used to assess whether drug-related effects observed during facial expression processing may reflect global effects of Epo on baseline cerebral blood flow. A flashing checkerboard (frequen$\mathrm{cy}=8 \mathrm{~Hz}$ ) was presented in blocks of $15 \mathrm{~s}$ alternating with $15 \mathrm{~s}$ of a fixation cross for a total of 20 cycles. Participants were instructed to lie with their eyes open during this control task. 


\section{fMRI Data Acquisition}

Imaging data were collected using a Siemens Sonata scanner operating at $1.5 \mathrm{~T}$, at the Oxford Centre for Clinical Magnetic Resonance Research. Functional imaging consisted of $35 \mathrm{~T} 2^{*}$-weighted echo-planar image slices (repetition time $(\mathrm{TR})=3000 \mathrm{~ms}$, echo time $(\mathrm{TE})=54 \mathrm{~ms}$, matrix $=128 \times 128), 1.5 \times 1.5 \times 4.5 \mathrm{~mm}^{3}$ voxels. To facilitate later co-registration of the fMRI data into standard space, we also acquired a Turbo FLASH sequence $(\mathrm{TR}=12 \mathrm{~ms}$, $\mathrm{TE}=5.65 \mathrm{~ms}$ ) voxel size $=1 \mathrm{~mm} 3$. The first two EPI images in each session were discarded to avoid $\mathrm{T} 1$ equilibration effects.

\section{fMRI Data Analysis}

fMRI data were pre-processed and analyzed using FSL version $3.2 \beta$ (www.fmrib.ox.ac.uk/fsl). After realignment, spatial normalization, and spatially smoothing (Gaussian kernel, $5 \mathrm{~mm}$ ), the time series in each session were highpass filtered (maximum of $0.04 \mathrm{~Hz}$ ). FSL was used to compute individual subject analyses in which the time series were prewhitened to remove temporal autocorrelation (Woolrich et al, 2001). Three experimental conditions were modeled; fear, happy, and neutral. Each condition was modeled separately by convolving trials with a canonical hemodynamic response function (Boynton et al, 1996). Temporal derivatives were included as covariates of no interest to increase statistical sensitivity. All analyses at the group level employed a full mixed effects approach (Woolrich et al, 2004). Z (Gaussian T) statistic images were thresholded using clusters determined at $Z=2.0$ and a corrected cluster significance of $P=0.05$. Foci of activation were localized with the aid of a standard anatomical atlas (Talairach and Tournoux, 1988). The following contrasts were chosen to maximize sensitivity to a drug effect on emotion processing: covert fear-covert neutral, covert happy-covert neutral, overt fear-overt neutral, and overt happy-overt neutral. For regions where significant drug group by emotion interactions were observed in the wholebrain analysis, mean percent BOLD signal change was extracted and examined with analysis of variance. Significant interactions were explored further with simple main effect analyses to identify the profile of drug effect. To assess amygdala response during faces processing, ROIs for the left and right amygdala in standard space were obtained using mri3dX (http://www.aston.ac.uk/lhs/staff/singhkd/ $\mathrm{mri3dX} / \mathrm{mri3dX}$.jsp), which utilizes a stored representation of the Talairach Daemon Database (Lancaster et al, 2000). Mean percent BOLD signal change during these conditions was computed in left and right amygdala and compared between Epo and placebo groups. For the control stimulation paradigm, we compared mean percent BOLD signal change in volunteers given Epo $v s$ placebo within a region of the occipital (calcarine) cortex activated by photic stimuli (Maldjian et al, 2003).

\section{Facial Expression Recognition}

Facial expression recognition was assessed using a different set of faces stimuli pictures also taken from Ekman and Friesen (1976), which were presented sequentially on a computer screen in a randomized order for $500 \mathrm{~ms}$. Faces expressed one of six basic emotions: happiness, surprise, sadness, fear, anger, and disgust, and participants were instructed to determine the particular emotions by pressing the corresponding keys on a keyboard as quickly and accurately as possible. Emotional expressions had been morphed between two standard images $0 \%$ (neutral) and $100 \%$ (full emotion) by taking a variable percentage of the shape and texture differences between the two in $10 \%$ steps (for details see Harmer et al, 2004). Four examples of every emotion at each intensity level were presented, and every face was also given in a neutral expression, making a total of 250 stimuli presentations. Accuracy, RT for correct choices, and misclassifications were recorded.

\section{Subjective Ratings}

On the day of drug administration, baseline mood was assessed using the Beck Depression Inventory (BDI; Beck et $a l, 1961$ ) and State-Trait Anxiety Inventory (STAI; Spielberger, 1983). Transient subjective state was assessed with Befindlichkeits Scale (BFS; Von Zerssen et al, 1974) and Visual Analog Scales (VAS) of relevant mood states at times $-15 \mathrm{~min}$, $+15 \mathrm{~min}$, $+60 \mathrm{~min}$, and $+120 \mathrm{~min}$ pre- and post-injection. Mood was measured with daily ratings on the PANAS (Watson et al, 1988), BFS, STAI, and VAS for 3 days after drug administration. On the test day, baseline mood was assessed with the BDI and transient subjective state with the BFS, STAI, and VAS at baseline, $+150 \mathrm{~min}$, and $+240 \mathrm{~min}$.

\section{Statistical Analysis of Behavioral and Mood Data}

Behavioral data and mood ratings were analyzed using repeated measures analysis of variance with group as the between-subjects factor and time as the within-subjects factor. Significant interactions were examined with simple main effect analyses. To obtain a further measure of accuracy for facial expression recognition corrected for the subject's response tendency, signal detection theory was applied. The proportion of correctly recognized stimuli (cr) and falsely recognized stimuli (fr) constitute the parametric sensitivity measure: $d^{\prime}=0.5((\mathrm{cr}-\mathrm{fr})(1+\mathrm{cr}-\mathrm{fr}) / 4 \mathrm{cr}(1-\mathrm{fr}))$, with a higher $d$-value reflecting greater recognition accuracy (Grier, 1971). All statistical analyses were performed using the statistical package for social sciences (SPSS).

\section{RESULTS}

\section{Biological Tests}

Consistent with previous reports (Miskowiak et al, in press), a single dose of Epo produced no significant changes in $\mathrm{Hb}(P>0.1)$ or hematocrit $(P>0.1)$, although there was a non-significant trend for RCC to be increased following Epo $v s$ placebo $(P=0.08$; Table 1$)$. However, this effect seems to be partly driven by decreased RCC in the placebo group and there was no significant difference between the baseline and post-treatment measurement in the volunteers receiving Epo $(P>0.2)$. Further, recordings of diastolic and systolic blood pressure on the test day revealed no differences between groups $(P>0.2)$. 
Table I Hematological Parameters for Volunteers Given Epo and Placebo

\begin{tabular}{|c|c|c|c|c|c|c|c|c|c|c|c|c|}
\hline & \multicolumn{4}{|c|}{ Hemoglobin (Hb) } & \multicolumn{4}{|c|}{ Hematocrit } & \multicolumn{4}{|c|}{ Red cell count } \\
\hline & \multicolumn{2}{|c|}{ Before } & \multicolumn{2}{|c|}{ After } & \multicolumn{2}{|c|}{ Before } & \multicolumn{2}{|c|}{ After } & \multicolumn{2}{|c|}{ Before } & \multicolumn{2}{|c|}{ After } \\
\hline & Mean & SD & Mean & SD & Mean & SD & mean & SD & Mean & SD & Mean & SD \\
\hline Epo $(N=12)$ & 14.5 & 1.4 & 14.8 & 1.0 & 0.43 & 0.04 & 0.44 & 0.03 & 4.95 & 0.48 & 5.04 & 0.33 \\
\hline Placebo $(N=12)$ & 14.7 & 1.5 & 14.5 & 1.3 & 0.44 & 0.04 & 0.43 & 0.03 & 4.99 & 0.41 & 4.87 & 0.32 \\
\hline Interaction & \multicolumn{4}{|c|}{$F(1.21)=2.9, P=0.10$} & \multicolumn{4}{|c|}{$\mathrm{F}(1,21)=2.7, P=0.11$} & \multicolumn{4}{|c|}{$F(I, 2 I)=3.5 P=0.08$} \\
\hline
\end{tabular}

A follow-up blood test for one volunteer allocated to placebo was lost and analysis therefore carried out for 23 volunteers of whom 12 had received Epo.

\section{Mood Ratings}

Groups were well matched in terms of general mood on both test occasions (all $P>0.5$ ). There were no acute effects of Epo on subjective state within the $2 \mathrm{~h}$ after administration (all $P>0.1$ ). On the day of the fMRI scan, there was no difference between groups in transient subjective state during testing (all $P>0.1$ ), allowing for examination of the effects of Epo on neural and cognitive processing of emotional information unconfounded by changes in mood, arousal, and anxiety.

Daily ratings of mood demonstrated no differences between groups on the STAI and VAS. In contrast, Epotreated volunteers scored significantly higher on positive affect on the PANAS for all 3 days compared with volunteers given placebo (main effect of group: $\mathrm{F}(1,22)=4.7, P=0.04)$, reflecting increased enthusiasm, energy, and alertness (Watson et al, 1988). Ratings on the BFS showed a general reduction in scores on total $(\mathrm{T})$ and energy (E) items (main effect of time: $\mathrm{F}(2,42)=4.6, P=0.02$ and $\mathrm{F}(2,42)=4.8, P=0.01$, respectively). In particular, Epotreated volunteers scored significantly lower on both $\mathrm{T}$ and $\mathrm{E}$ items than those given placebo (main effect of group: $\mathrm{T}: \mathrm{F}(1,21)=9.4, P=0.006, \mathrm{E}: \mathrm{F}(1,21)=9.0, P=0.007)$, suggesting increased well-being and energy levels.

\section{BOLD Response during Facial Expression Processing}

Main effect of task. Overt presentations: Presentations of overt fearful and happy expressions produced significant bilateral amygdala activation $(\mathrm{F}(1,23)=18.9, P<0.001$, and $\mathrm{F}(1,23)=16.4, P=0.001$, respectively), as expected. In the whole brain analysis, overt fearful, happy, and neutral expressions activated a similar network of frontal, temporal, cingulate, parietal, and occipital regions to those reported previously (eg Miskowiak et al, in press) (for peak cluster activation, see Table 2).

Covert presentations: In contrast to overt presentations, the amygdala was not significantly activated in the masked fear condition $(P>0.4)$. Covert presentations of fearful, happy, and neutral faces activated a broad neural network of regions including middle, inferior, and superior frontal gyri, posterior cingulate, middle and inferior temporal gyrus, and parietal and occipital cortices (for peak cluster activation in these regions, see Table 2 ).

Epo $\times$ task interactions. Overt presentations: ROI analysis revealed that Epo significantly increased left amygdala
BOLD signal change during overt presentations of fearful, happy, and neutral faces compared with placebo (main effect of group: $F(1,22)=5.03, P=0.04$; Figure 1). There were no other effects of Epo in the whole brain analysis for the unmasked condition (all $P>0.05$, corrected).

Covert presentations: Consistent with the absence of amygdala response to masked fearful faces, there was no effect of Epo on amygdala signal during this condition. In the whole-brain analysis, the interaction between emotion condition and drug group revealed increased neural response under Epo $v s$ placebo to masked fearful $v s$ neutral faces in a cluster in the right parietal cortex including the precuneus and inferior and superior parietal gyrus (Figure 2a) and masked happy vs neutral faces in two clusters in the bilateral precunei and left cuneus (Figure 2b). Extraction of signal change in these regions demonstrated that Epo specifically increased response to masked presentations of fear $(\mathrm{F}(1,22)=21.8, P<0.001$; $t=2.7, \mathrm{df}=22, P=0.02$; Figure $2 \mathrm{a})$ and happiness $(\mathrm{F}(1,22)=$ 17.7; $P<0.001 ; t=3.9, \mathrm{df}=22, P=0.001$; Figure $2 \mathrm{~b})$. The effects of Epo on neuronal responses occurred in absence of any group differences in accuracy $(P>0.4)$ or RT $(P>0.1)$ of gender discrimination.

There were no other effects of task or Epo in the whole brain analysis reported for both the overt and covert conditions.

\section{Visual Stimulation Control Experiment}

Epo did not significantly influence mean percent BOLD signal change to photic stimulation in the region of occipital (calcarine) cortex normally activated by photic stimulation ( $t=0.8, \mathrm{df}=22, P=0.43)$. The observed effects during faces processing are therefore likely to reflect task-specific rather than global hemodynamic changes.

\section{Facial Expression Recognition}

Epo improved overall accuracy of facial expression recognition (main effect of group: $\mathrm{F}(1,22)=5.2, P=0.03$ ) in absence of any effect on RTs $(P>0.05)$. Signal detection analysis demonstrated greater discrimination accuracy for all emotions in Epo-treated participants compared with those given placebo (main effect of group: $F(1,22)=4.6$, $P=0.04)$ in absence of any group differences in response biases (all $P>0.7$ ).

Investigation of facial expression recognition over the different morphed intensity levels revealed that Epo improved recognition of lower levels of happiness (inter- 
Table 2 Peak Cluster Activation in Brain Regions of Significantly Increased BOLD Response during Facial Expression Processing in PlaceboTreated Volunteers (Main Effect of Task)

\begin{tabular}{|c|c|c|c|c|c|c|c|c|c|}
\hline \multirow[b]{2}{*}{ Task and Region } & \multirow[b]{2}{*}{ Z-value } & \multicolumn{3}{|c|}{ Coordinates } & \multirow[b]{2}{*}{ Task and Region } & \multirow[b]{2}{*}{ Z-value } & \multicolumn{3}{|c|}{ Coordinates } \\
\hline & & $\mathbf{x}$ & $\mathbf{Y}$ & $\mathbf{Z}$ & & & $\mathbf{x}$ & $\mathbf{Y}$ & $\mathbf{Z}$ \\
\hline L precentral gyrus (BA 4) & 3.44 & -22 & -10 & 50 & L medial frontal gyrus (BA 6) & 6.67 & 2 & 2 & 58 \\
\hline L superior frontal gyrus (BA 6) & 4.63 & -6 & 4 & 58 & R precentral gyrus (BA 6) & 4.49 & 46 & 0 & 32 \\
\hline \multirow{2}{*}{ L putamen } & & & & & $\mathrm{R}$ cerebellum, declive & 9.25 & 40 & -60 & -16 \\
\hline & & & & & L putamen & 4.05 & -24 & 6 & 4 \\
\hline Covert happy faces & & & & & Overt happy faces & & & & \\
\hline Left superior frontal gyrus (BA 6) & 4.15 & -8 & 4 & 60 & L medial frontal gyrus (BA 6) & 3.44 & -44 & 0 & 38 \\
\hline Covert neutral faces & & & & & Overt neutral faces & & & & \\
\hline R inferior frontal gyrus (BA 45) & 4.79 & 34 & 24 & 10 & L medial frontal gyrus (BA 6) & 3.65 & 28 & 0 & 0 \\
\hline R superior frontal gyrus (BA 6) & 3.68 & 4 & 6 & 58 & R precentral gyrus (BA 6) & 4.42 & 46 & 2 & 32 \\
\hline L superior parietal lobule (BA 7) & 5.3 & -28 & -64 & 52 & L inferior parietal lobule (BA 40) & 5.28 & -44 & -28 & 44 \\
\hline \multirow[t]{4}{*}{ L lingual gyrus (BA |8) } & 6.74 & -4 & -80 & 0 & R inferior parietal gyrus (BA 40) & 3.84 & -22 & -44 & 42 \\
\hline & & & & & R fusiform gyrus (BA 19) & 6.58 & 40 & -66 & -8 \\
\hline & & & & & L putamen & 5.33 & 0 & 2 & 62 \\
\hline & & & & & R putamen & 4.42 & 46 & 2 & 32 \\
\hline
\end{tabular}

BA, Brodmann area.

$\mathrm{MNI}$ coordinates $(x, y, z)$ refer to peak activation within each cluster identified thresholded at $Z=2.0$ and $P<0.05$, corrected.

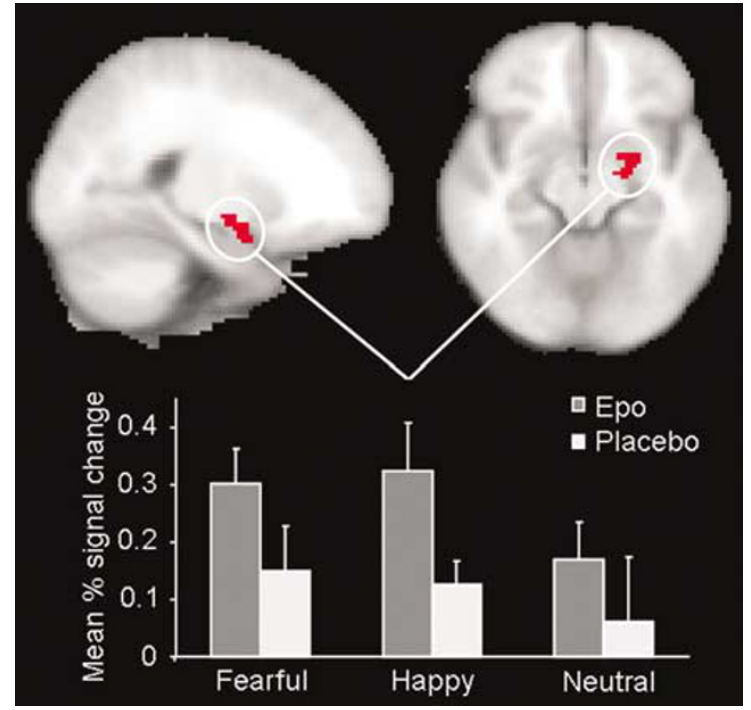

Figure I Differences in left amygdala response to overt presentations of fearful, happy, and neutral faces in the volunteers treated with Epo vs placebo. Epo $(N=\mid I)$ enhanced left amygdala BOLD signal change to all facial expressions vs baseline compared to placebo $(N=12)$ (ANOVA; main effect of group $F(1,22)=5.03, P=0.035)$. Values represent mean percent signal change. Error bars represent the standard error (SEM). action intensity $\times$ group: $\mathrm{F}(9,198)=2.1, P=0.03$; Figure 3 ) and fear (interaction intensity $\times$ group: $\mathrm{F}(9,198)=2.6$, $P=0.007$; Figure 3). Effects on the recognition of other emotions across the morphed intensity levels were not seen (all $P>0.2$ ).

\section{DISCUSSION}

The current study demonstrated that a single intravenous administration of Epo (40000 IU) vs saline to healthy volunteers enhanced the neural and cognitive processing of facial expressions on day 3 post-administration and improved self-reported mood in the 3 day period before the scan. These effects are likely to reflect direct neurobiological actions of Epo in absence of marked changes in red cell mass in the experimental group.

\section{Epo Improves Self-Reported Mood}

The current study replicated our previous finding of improved self-reported mood for 3 days following Epo vs saline administration to healthy volunteers (Miskowiak et al, in press). Epo-treated volunteers had significantly higher positive affect scores on the PANAS than those given 


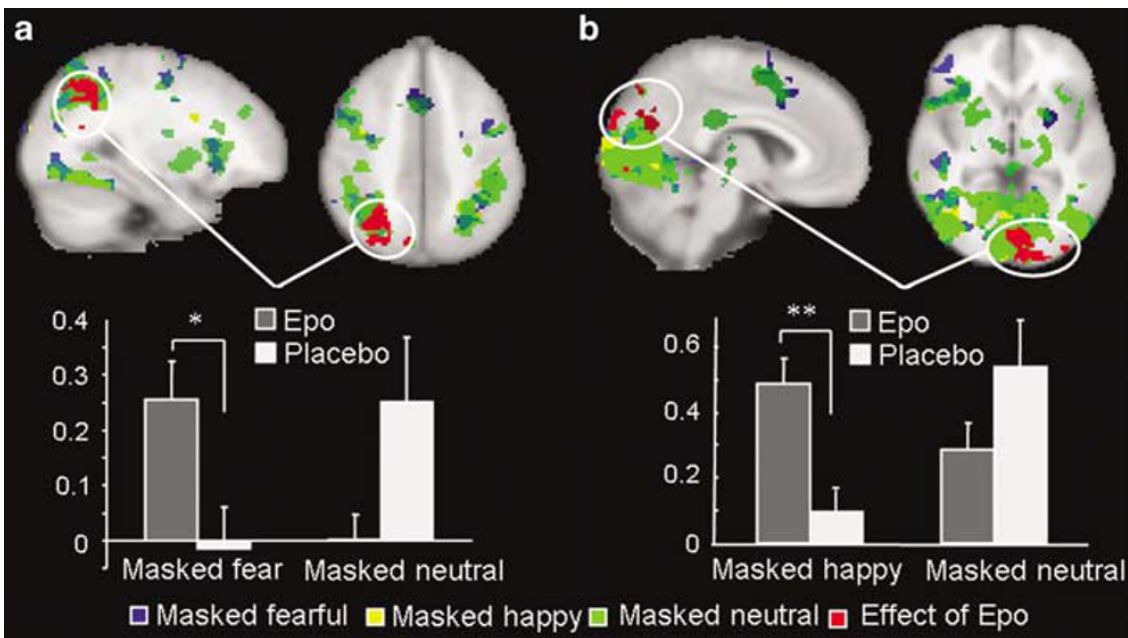

Figure 2 (a) Neuronal responses to masked fearful and happy vs neutral faces. Areas marked with blue, yellow, and green are the regions significantly activated by masked fearful, happy, and neutral faces, respectively. Epo increased response to masked fearful vs neutral faces in a cluster encompassing the right parietal cortex (areas marked with red). Plot of mean percent BOLD signal change to fearful and neutral faces modeled against baseline (fixation periods) under Epo (dark bars) and placebo (light bars) in these regions of significant drug group (Epo, placebo) $\times$ emotion (fearful, neutral) interaction. (b) Epo increased response to masked happy vs neutral faces in bilateral precuneui and left cuneus (areas marked with red). Plot of mean percent BOLD signal change to happy and neutral faces modeled against baseline (fixation periods) under Epo (dark bars) and placebo (light bars) in the region of significant drug group (Epo, placebo) $\times$ emotion (happy, neutral) interaction. Images are thresholded at $Z=2.0, P=0.05$, corrected, and represent a radiological view of the brain. Bars show the mean; error bars show the standard error (SEM). $* P<0.05$.
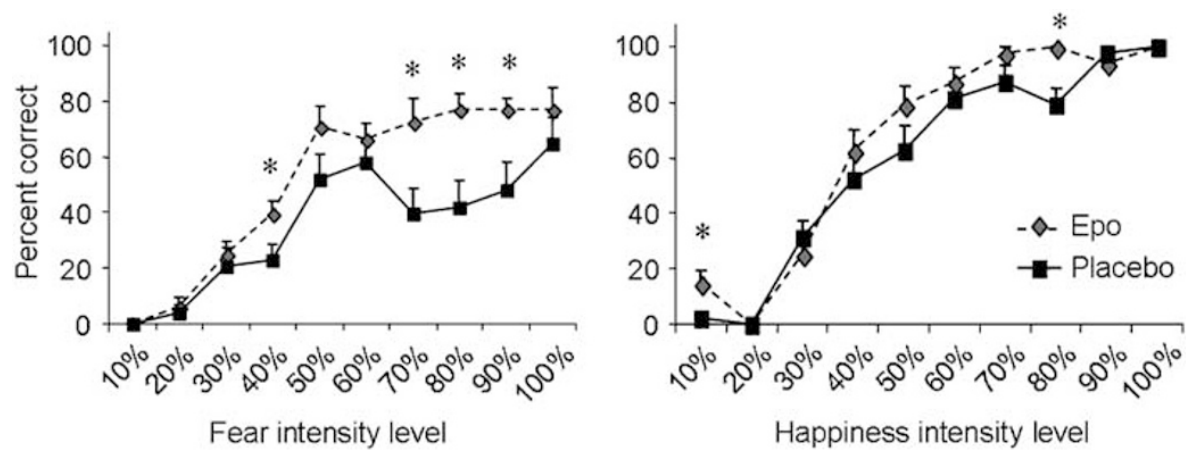

Figure 3 Recognition of fear and happiness across the different intensity levels from 10\% to 100\% (full emotion). Volunteers given Epo (dashed line) demonstrated increased recognition of fearful facial and happy expressions compared to placebo (solid line) (interaction group $\times$ emotion intensity fear: $F(9,198)=2.6, P=0.007$, and happiness: $F(9,198)=2.1, P=0.03)$. Values represent mean accuracy \pm I SEM $* P<0.05$.

placebo as well as increased well being and energy on the BFS. Our previous study suggested reduced negative rather than increased positive affect, so we cannot say whether Epo affects both of these processes or whether the balance of the mood improvement interacts with trait or state characteristics of the sample. Nonetheless, this rapid improvement of mood, which persisted for 3 days post-Epo-administration, is noteworthy and deserves further investigation.

\section{Epo Enhances Neural and Cognitive Processing of Faces on Day 3}

Although a number of human imaging studies suggest a specific role of amygdala in the processing of threat-related signals (eg Morris et al, 1996; Phillips et al, 1998), the link between amygdala and threat is not exclusive. This is highlighted by the activation of amygdala by presentations of unfamiliar faces (Dubois et al, 1999), facial display of sadness (Blair et al, 1999) and happiness (Breiter et al, 1996), as well as emotive scenes (Taylor et al, 2000) and film extracts (Reiman et al, 1997). The recent demonstration of left amygdala response not only to fearful but also to disgusted, angry, sad, neutral, and happy faces indicates a general purpose function of amygdala in the processing of salient information from faces (Fitzgerald et al, 2006). The increased left-hemisphere amygdala response to overt fearful, happy, and neutral faces in volunteers given Epo vs placebo is thus consistent with increased vigilance for facial expressions irrespective of valence.

Although amygdala activation has been reported with subliminal presentations of fear (Whalen et al, 1998), this effect is not uniformly seen (eg Phillips et al, 2004). In our sample, covert presentations did not activate the amygdala, which may explain the absence of an effect of Epo on amygdala response during this condition. In contrast, Epo significantly enhanced occipito-parietal response to covert happy $v s$ neutral faces and parietal activation to covert fearful $v s$ neutral faces. These regions were partly overlapping with those activated by covert presentations of fearful and happy faces in placebo-treated volunteers (see 
Figure 2), suggesting that Epo modulates faces-relevant to neural processing. A general increase in occipito-parietal activation to fearful $v s$ happy faces in healthy volunteers is thought to be a mechanism by which attentional resources are captured by threatening stimuli (Pourtois et al, 2006). The increased occipito-parietal activation to happy faces and increased parietal activation to fearful faces could therefore suggest that Epo increased subliminal vigilance towards facial displays of emotion in general.

The effects of Epo on neural processing of faces were accompanied by improvement in overall recognition accuracy for facial expressions of fear, happiness, disgust, anger, surprise and sadness after the scan. In particular, Epo-treated volunteers displayed enhanced recognition of lower intensity levels of happiness and fear, similar to acute effects of serotonergic antidepressants (Harmer et al, 2003). By contrast, we reported previously a decrease in fear recognition and neural response to fearful faces 1 week after administration of Epo (Miskowiak et al, in press). This reversal of effect is strikingly similar to our previous findings with SSRI administration in healthy volunteers (eg Harmer et al, 2003, 2006), where increased fear recognition is seen with acute administration but after 7 days of treatment, volunteers show a reduction in this aspect of fear processing relative to matched placebo treatment. While the mechanisms mediating this reversal are unknown, the current finding suggests that Epo taps in to emotion processing in ways that resemble conventional antidepressants; early increases in the processing of happy facial expressions accompanied by a reversal of increased to decreased fear processing over time.

\section{Limitations}

A limitation of pharmacological fMRI studies in general is the possibility that drug effects on neural response may be confounded by non-specific effects on neural coupling and cerebral hemodynamics (Bonne et al, 1999). There is evidence of a positive correlation between blood pressure and BOLD response (Wang et al, 2006), which could confound data interpretation. While repeated administration of Epo may increase blood pressure in response to elevated red cell mass, we found no significant differences between groups in blood pressure on the day of scanning. In addition, Epo had no effect on neural response to flashing checker-board stimuli in the region of primary visual cortex normally activated by photic stimulation. It is therefore unlikely that the observed effects of Epo on neural response during faces processing resulted from non-specific hemodynamic changes.

It is possible that the effects of Epo reported here on the neural and cognitive processing of facial expressions occurred as an indirect effect of the increased mood and arousal seen in this group rather than a direct neurobiological action on the underlying neural processes. However, by the day of the scan, ratings of mood and arousal had returned to normal and were indistinguishable from the placebo-treated participants. In future studies, however, more detailed examination of the mood and arousal changes seen following Epo and their relation to changes in emotional processing observed 3 days post-administration should be performed.
Finally, although the present findings suggest that Epo influences cognitive and neural function and mood in ways compatible with an antidepressant action in healthy volunteers, the clinical effect in patients suffering from depression is unknown. If changes in the processing of emotional information and improvement of mood are important in the therapeutic actions of antidepressant drugs as hypothesized (Harmer et al, 2004), then Epo may have some benefit in these patients. However, we have also hypothesized that acute increase in fear processing may relate to anxiogenic effects of SSRIs seen early in treatment (Harmer et al, 2003). The possibility of such effects should therefore also be considered in clinical studies investigating the effects of Epo.

\section{CONCLUSIONS}

In conclusion, the present study showed that Epo influences neuronal and cognitive function in healthy volunteers 3 days after administration. Epo influenced neural and cognitive processing of emotional facial expressions in ways similar to the actions of serotonergic antidepressants. The replication of mood improvement for 3 days following Epo administration is also noteworthy given the time to effect of conventional antidepressant drug treatments. Together, these findings highlight Epo as a candidate for neurotrophic add-on treatment and suggest that characterization of the effects in a clinically depressed group would be of great potential interest.

\section{ACKNOWLEDGEMENTS}

This study was supported by the Lundbeckfonden, Denmark, the ESRC, UK, and the Goodger Scholarship, UK.

\section{DISCLOSURE/CONFLICT OF INTEREST}

The authors declare the following potential conflicts of interest: Dr Wise has received compensation from GlaxoSmithKline over the past 3 years. Professor Goodwin has received grants from Sanofi-Aventis and Servier, honoraria from AstraZeneca, BMS, Eisai, Lundbeck, Sanofi-Aventis, and Servier, and is on the Advisory board for BMS, Lilly, Lundbeck, P1Vital, Sanofi-Aventis, Servier, and Wyeth. Dr Harmer has acted as a consultant for Lundbeck, Merck, Sharpe, Dohme, and P1Vital.

\section{REFERENCES}

Beck AT, Ward CH, Mendelssohn MJ, Erbaugh J (1961). An inventory for measuring depression. Arch Gen Psychiatry 4: 561-571.

Berton O, Nestler E (2006). New approaches to antidepressant drug discovery: beyond monoamines. Nat Neurosci 7: 137-148.

Blair RJ, Morris JS, Frith CD, Perrett DI, Dolan RJ (1999). Dissociable neural responses to facial expressions of sadness and anger. Brain 122: 883-893.

Bonne O, Krausz Y, Aharon Y, Gelfin Y, Chisin R, Lerer B (1999). Clinical doses of fluoxetine and cerebral blood flow in healthy volunteers. Psychopharmacology 143: 24-28.

Boynton GM, Engel SA, Glover GH, Heeger DJ (1996). Linear systems analysis of functional magnetic resonance imaging in human V1. J Neurosci 16: 4207-4221. 
Breiter HC, Etcoff NL, Whalen PJ, Kennedy WA, Rauch SL, Buckner RL et al (1996). Response and habituation of the human amygdala during visual processing of facial expression. Neuron 17: 875-887.

Brines M, Cerami A (2005). Emerging biological roles for erythropoietin in the nervous system. Nat Neurosci 6: 484-494.

Brines ML, Ghezzi P, Keenan S, Agnello D, de Lanerolle NC, Cerami C et al (2000). Erythropoietin crosses the blood-brain barrier to protect against experimental brain injury. Proc Natl Acad Sci USA 97: 10526-10531.

Dubois S, Rossion B, Schiltz C, Bodart JM, Michel C, Bruyer R, Crommelinck M (1999). Effect of familiarity on the processing of human faces. Neuroimage 9: 278-289.

Ehrenreich H, Hasselblatt M, Dembowski C, Cepek L, Lewczuk P, Stiefel $\mathrm{M}$ et al (2002). Erythropoietin therapy for acute stroke is both safe and beneficial. Mol Med 8: 495-505.

Ehrenreich H, Hinze-Selch D, Stawicki S, Aust C, Knolle-Veentjer S, Wilms $S$ et al (2007). Improvement of cognitive function in chronic schizophrenic patients by recombinant human erythropoietin. Mol Psychiatry 1-15.

Ekman P, Friesen WV (1976). Pictures of Facial Affect. Consulting Psychologists Press: Palo Alto, CA.

Fitzgerald DA, Angstadt M, Jelsone LM, Nathan PJ, Phan KL (2006). Beyond threat: amygdala reactivity across multiple expressions of facial affect. Neuroimage 30: 1441-1448.

Grier JB (1971). Nonparametric indices for sensitivity and bias: computing formulas. Psychol Bull 75: 9424-9429.

Hariri AR, Goldberg TE, Mattay VS, Kolachana BS, Callicott JH, Egan MF, Weinberger DR (2003). Brain-derived neurotrophic factor val66met polymorphism affects human memory-related hippocampal activity and predicts memory performance. J Neurosci 23: 6690-6694.

Harmer CJ, Bhagwagar Z, Perrett DI, Vollm BA, Cowen PJ, Goodwin GM (2003). Acute SSRI administration affects the processing of social cues in healthy volunteers. Neuropsychopharmacology 28: 148-152.

Harmer CJ, Mackay CE, Reid CB, Cowen PJ, Goodwin GM (2006). Antidepressant drug treatment modifies the neural processing of nonconscious threat cues. Biol Psychiatry 59: 816-820.

Harmer CJ, Shelley NC, Cowen PJ, Goodwin GM (2004). Increased positive versus negative affective perception and memory in healthy volunteers following selective serotonin and norepinephrine reuptake inhibition. Am J Psychiatry 161: 1256-1263.

King CE, Rodger J, Bartlett C, Esmaili T, Dunlop SA, Beazley LD (2007). Erythropoietin is both neuroprotective and neuroregenerative following optic nerve transection. Exp Neurol [E-pub ahead of print].

Knutson B, Wolkowitz OM, Cole SW, Chan T, Moore EA, Johnson RC et al (1998). Selective alteration of personality and social behavior by serotonergic intervention. Am J Psychiatry 155: 373-379.

Lancaster JL, Woldorff MG, Parsons LM, Liotti M, Freitas CS, Rainey L et al (2000). Automated Talairach atlas labels for functional brain mapping. Hum Brain Mapp 10: 120-131.

Leitgeb C, Pecherstorfer M, Fritz E, Ludwig H (1994). Quality of life in chronic anemia of cancer during treatment with recombinant human erythropoietin. Cancer 73: 2535-2542.

Maldjian JA, Laurienti PJ, Kraft RA, Burdette JH (2003). An automated method for neuroanatomic and cytoarchitectonic atlas-based interrogation of fMRI data sets. Neuroimage 19: $1233-1239$.

Miskowiak K, O’Sullivan U, Harmer C (2007). Erythropoietin enhances hippocampal response during memory retrieval in humans. J Neurosci 27: 2788-2792.

Miskowiak K, O'Sullivan U, Harmer C. Erythropoietin reduces neural and cognitive processing of fear in human models of antidepressant drug action (in press).
Morris JS, Frith CD, Perrett DI, Rowland D, Young AW, Calder AJ, Dolan RJ (1996). A differential neural response in the human amygdala to fearful and happy facial expressions. Nature 383: 812-815.

Nissenson AR (1992). Epoetin and cognitive function. Am J Kidney Dis XX: 21-24.

Phillips ML, Williams LM, Heining M, Herba CM, Russell T, Andrew C et al (2004). Differential neural responses to overt and covert presentations of facial expressions of fear and disgust. Neuroimage 21: 1484-1496.

Phillips ML, Young AW, Scott SK, Calder AJ, Andrew C, Giampietro V et al (1998). Neural responses to facial and vocal expressions of fear and disgust. Proc R Soc Lond B Biol Sci 265: 1809-1817.

Pourtois G, Schwartz S, Seghier ML, Lazeyras F, Vuilleumier P (2006). Neural systems for orienting attention to the location of threat signals: an event related fMRI study. Neuroimage 31: 920-933.

Reiman EM, Lane RD, Ahern GL, Schwartz GE, Davidson RJ, Friston KJ et al (1997). Neuroanatomical correlates of externally and internally generated human emotion. Am J Psychiatry 154: 918-925.

Santarelli L, Saxe M, Gross C, Surget A, Battaglia F, Dulawa S et al (2003). Requirement of hippocampal neurogenesis for the behavioral effects of antidepressants. Science 301: 805-809.

Shirayama Y, Chen AC, Nakagawa S, Russell DS, Duman RS (2002). Brain-derived neurotrophic factor produces antidepressant effects in behavioral models of depression. J Neurosci 22: 3251-3261.

Siren AL, Fratelli M, Brines M, Goemans C, Casagrande S, Lewczuk $\mathrm{P}$ et al (2001). Erythropoietin prevents neuronal apoptosis after cerebral ischemia and metabolic stress. Proc Natl Acad Sci USA 98: 4044-4049.

Spielberger CD (1983). Manual for the State-Trait Anxiety Inventory (STAI). Consulting Psychologists Press: Palo Alto, CA.

Talairach J, Tournoux P (1988). Co-planar Stereotaxic Atlas of the Human Brain. Thieme: New York.

Taylor SF, Liberzon I, Koeppe RA (2000). The effect of graded aversive stimuli on limbic and visual activation. Neuropsychologia 38: 1415-1425.

Viviani B, Bartesaghi S, Corsini E, Villa P, Ghezzi P, Garau A et al (2005). Erythropoietin protects primary hippocampal neurons increasing the expression of brain-derived neurotrophic factor. J Neurochem 93: 412-421.

Von Zerssen D, Strain F, Schwartz D (1974). Evaluation of depressive states, especially in longitudinal studies. In: Pichot $\mathrm{P}$ (ed). Psychological Measurement in Psychopharmacology, Modern Problems in Pharmacopsychiatry. Karger: Basle. pp 189-202.

Wang L, Zhang Z, Wang Y, Zhang R, Chopp M (2004). Treatment of stroke with erythropoietin enhances neurogenesis and angiogenesis and improves neurological function in rats. Stroke 35: 1732-1737.

Wang R, Foniok T, Wamsteeker JI, Qiao M, Tomanek B, Vivanco RA, Tuor UI (2006). Transient blood pressure changes affect the functional magnetic resonance imaging detection of cerebral activation. Neuroimage 31: 1-11.

Watson D, Clark LA, Tellegen A (1988). Development and validation of brief measures of positive and negative affect: the PANAS scales. J Pers Soc Psychol 54: 1063-1070.

Whalen PJ, Rauch SL, Etcoff NL, McInerney SC, Lee MB, Jenike MA (1998). Masked presentations of emotional facial expressions modulate amygdala activity without explicit knowledge. J Neurosci 18: 411-418.

Woolrich MW, Behrens TE, Beckmann CF, Jenkinson M, Smith SM (2004). Multilevel linear modelling for FMRI group analysis using Bayesian inference. Neuroimage 21: 1732-1747.

Woolrich MW, Ripley BD, Brady M, Smith SM (2001). Temporal autocorrelation in univariate linear modeling of FMRI data. Neuroimage 14: 1370-1386. 